\title{
Ultrasonography with color Doppler and power Doppler in the diagnosis of periapical lesions
}

\author{
Sumit Goel, Suma Gundareddy Nagendrareddy ${ }^{1}$, Manthena Srinivasa Raju², \\ Dayashankara Rao Jingade Krishnojirao³, Rajul Rastogi4, Ravi Prakash Sasankoti Mohan5, Swati Gupta \\ Department of Oral Medicine and Radiology, Subharti Dental College and Hospital, Meerut, 'ITS Dental College, Muradnagar, \\ ${ }^{2}$ Teerthanker Mahaveer Dental College, Moradabad, Uttar Pradesh, ${ }^{3}$ Department of Oral and Maxillofacial Surgery, SGT Dental \\ College, Gurgaon, Haryana, ${ }^{4}$ Department of Radiodiagnosis, Yash Diagnostic Centre, Moradabad, ${ }^{5}$ Department of Oral Medicine \\ and Radiology, Kothiwal Dental College, Moradabad, Uttar Pradesh, India
}

Correspondence: Dr. Sumit Goel, Department of Oral Medicine and Radiology, Subharti Dental College and Hospital, Swami Vivekanand Subharti University, Meerut - 201201, Uttar Pradesh, India. E-mail: drsumitomr@gmail.com

\begin{abstract}
Aim: To evaluate the efficacy of ultrasonography (USG) with color Doppler and power Doppler applications over conventional radiography in the diagnosis of periapical lesions. Materials and Methods: Thirty patients having inflammatory periapical lesions of the maxillary or mandibular anterior teeth and requiring endodontic surgery were selected for inclusion in this study. All patients consented to participate in the study. We used conventional periapical radiographs as well as USG with color Doppler and power Doppler for the diagnosis of these lesions. Their diagnostic performances were compared against histopathologic examination. All data were compared and statistically analyzed. Results: USG examination with color Doppler and power Doppler identified 29 (19 cysts and 10 granulomas) of 30 periapical lesions accurately, with a sensitivity of $100 \%$ for cysts and $90.91 \%$ for granulomas and a specificity of $90.91 \%$ for cysts and $100 \%$ for granulomas. In comparison, conventional intraoral radiography identified only 21 lesions (sensitivity of $78.9 \%$ for cysts and $45.4 \%$ for granulomas and specificity of $45.4 \%$ for cysts and $78.9 \%$ for granulomas). There was definite correlation between the echotexture of the lesions and the histopathological features except in one case. Conclusions: USG imaging with color Doppler and power Doppler is superior to conventional intraoral radiographic methods for diagnosing the nature of periapical lesions in the anterior jaws. This study reveals the potential of USG examination in the study of other jaw lesions.
\end{abstract}

Key words: Color Doppler; conventional intraoral radiography; histopathology; periapical lesions; power Doppler; ultrasound

\section{Introduction}

Traditionally, the diagnosis of periapical lesions of the teeth has been based on clinical, radiological, and histopathological examinations..$^{[1]}$ Conventional radiological procedures have limitations as they do not allow differentiation between

\begin{tabular}{|l|l|}
\hline \multicolumn{2}{|c|}{ Access this article online } \\
\hline Quick Response Code: & \\
\hline & Website: \\
\hline & www.ijri.org \\
\cline { 2 - 2 } & DOI: \\
\hline
\end{tabular}

cystic and noncystic periapical lesions. ${ }^{[2,3]}$ In view of this, USG has been used recently in the field of endodontic diagnosis as it provides more detailed information. ${ }^{[2]}$

The number of studies investigating the role of USG in the diagnosis of periapical bone lesions of endodontic origin is limited. ${ }^{[4-6]}$ This research is an effort to evaluate the potential of USG with color Doppler and power Doppler for diagnosing periapical lesions in a large sample.

\section{Materials and Methods}

Thirty patients aged between 15 years and 50 years having endodontic problems in the maxillary or mandibular anterior teeth and with radiographic evidence of periapical radiolucency were selected for this study. 


\section{Conventional radiographic examination}

In all the 30 patients, periapical radiographs were made by the bisecting angle technique using the Snap-A-Ray ${ }^{\circledR}$ film holder (Gnatus dental X-ray unit; at $70 \mathrm{kVp}, 8 \mathrm{~mA}$, $0.7 \mathrm{~s}, 2.5 \mathrm{~mm} \mathrm{Al} /$ equiv filtration, $60-\mathrm{mm}$ beam diameter, and focal spot-to-skin distance of $200 \mathrm{~mm}$ ) and Kodak E-speed ${ }^{\circledR}$ films (No. 2, $31 \mathrm{~mm} \times 41 \mathrm{~mm}$ size) (Eastman Kodak Co., France). The presence of a periapical lesion was confirmed on the basis of the clinical and radiological findings. The dimensions of all 30 lesions were measured in a superoinferior and mesiodistal direction on a millimeter scale using calipers and a ruler.

Diagnosis was based on the following criteria: ${ }^{[7]}$

Periapical cyst: A well-circumscribed round or oval radiolucent periapical lesion with or without a sclerotic border and measuring more than $1 \mathrm{~cm}$ in diameter.

Periapical granuloma: A well-circumscribed radiolucent periapical lesion with or without a sclerotic border and measuring less than $1 \mathrm{~cm}$ in size.

\section{USG examination}

The lesion was then subjected to an USG examination using a SonoAce 8000 Live $^{\circledR}$ machine (Medison, Seoul, Korea) with a high-definition, linear, regular-size, multifrequency USG probe at a frequency of $9 \mathrm{MHz}$. Both transverse and longitudinal scans were obtained by placing the ultrasound probe extraorally as well as intraorally in the buccal sulcus overlying the apical area of the affected tooth. However, in some patients, intraoral scanning was not possible because the vestibule was too shallow.

Possible fenestration and thin anterior bone permitted USG images to be obtained in all 30 cases. The lesions were measured in all the three planes. Color Doppler and power Doppler were then applied to each examination. The USG diagnosis was based on the following criteria: $[5,6,8]$

Periapical cyst: A hypoechoic, well-contoured cavity surrounded by reinforced bone walls and filled with fluid, with no evidence of internal vasculature on color and power Doppler examinations.

Periapical granuloma: A poorly defined lesion, which was frankly corpusculated (hyperechoic/echogenic) or had both corpusculated and hypoechoic areas, and exhibiting a rich vascular supply on color and power Doppler examinations.

\section{Histopathological examination}

Biopsy samples were obtained from all the 30 patients through periapical surgery and specimens were sent for routine histopathological examination to confirm the diagnosis [Figures 1 and 2].

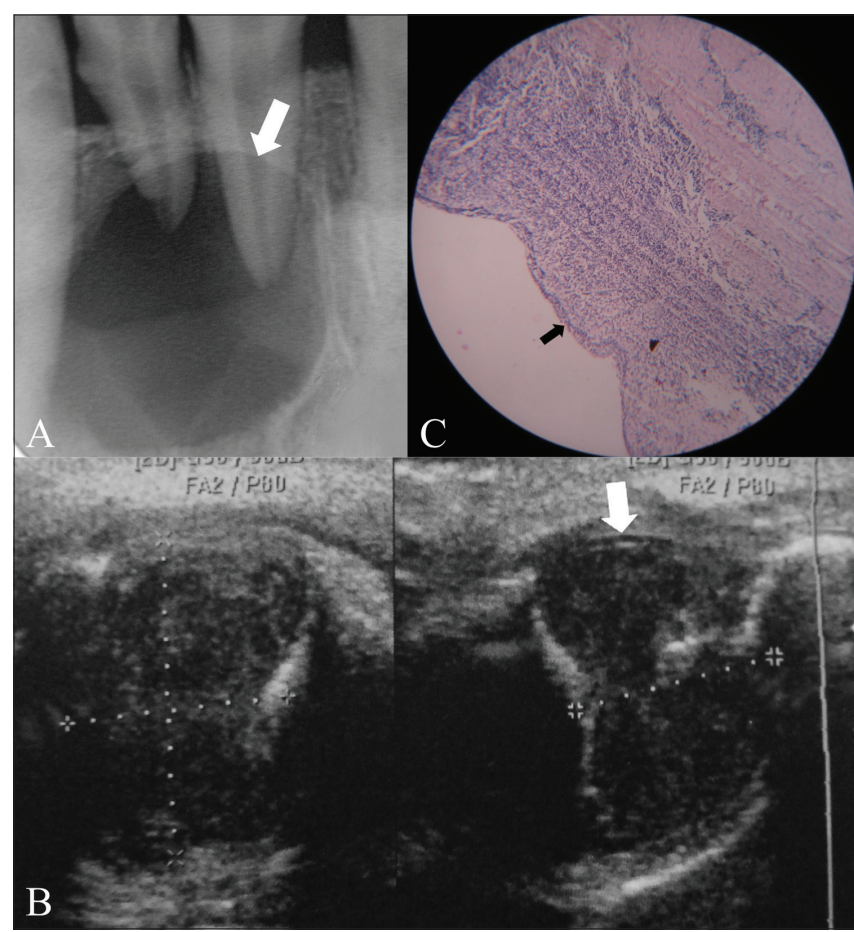

Figure 1 (A-C): Periapical cyst. Intraoral periapical radiograph (A) shows a well-circumscribed radiolucent periapical lesion (arrow) with a partial sclerotic border, measuring more than $1 \mathrm{~cm}$ in diameter. USG with color Doppler and power Doppler (B) shows a hypoechoic, well-contoured cavity (arrow) with no evidence of internal vasculature on color and power Doppler examinations. $\mathrm{H}$ and $\mathrm{E}$ stained section shows the presence of an epithelial lining (arrow) with underlying dense fibrocellular connective tissue stroma (arrowhead)

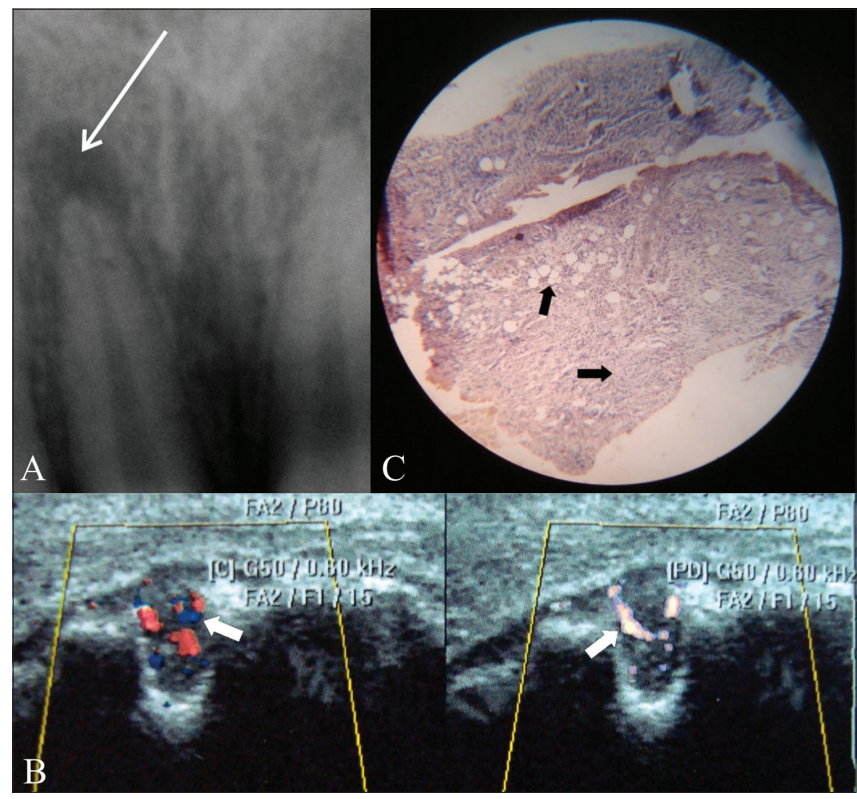

Figure 2 (A-C): Periapical granuloma. Intraoral periapical radiograph (A) shows a well-circumscribed radiolucent periapical lesion (arrow) without a sclerotic border, measuring less than $1 \mathrm{~cm}$ in size. USG with color Doppler and power Doppler shows a poorly defined hypoechoic lesion (arrow) exhibiting a rich vascular supply on color and power Doppler examinations. H\&E stained section (C) shows fibrocellular connective tissue stroma (arrow) consisting of chronic inflammatory cell infiltrate (mainly lymphocytes and plasma cells) and endothelium-lined blood capillaries with red blood cells, fibroblasts, and collagen fibers 


\section{Results}

\section{Conventional radiographic examination}

Intraoral radiographic examination showed a definite presence of periapical radiolucencies in all the 30 cases. Twenty-one radiolucent lesions with well-defined borders and with size $>1 \mathrm{~cm}$ were diagnosed as periapical cysts, while nine radiolucent lesions with well-defined borders and with a greatest diameter of $<1 \mathrm{~cm}$ were diagnosed as periapical granulomas. The size of the lesion was measured both superoinferiorly and mesiodistally. The mean of the superioinferior measurements was found to be $12.50 \mathrm{~mm}$ (standard deviation: 3.17 ) while the mean of the mesiodistal measurement was $11.33 \mathrm{~mm}$ (standard deviation: 4.32) [Table 1, Figure 3]

\section{USG examination}

USG examination easily identified all the 30 periapical lesions and showed discontinuity in the overlying cortical bone. Twenty of these were hypoechoic or anechoic lesions with well-defined smooth contours and no evidence of internal vascularization on application of both color Doppler and power Doppler. These were diagnosed as periapical cysts. Out of these 20 lesions, 13 showed floating internal echoes indicative of infected cyst, and 2 showed vascularity at the periphery of the lesion suggestive of a

Table 1: Summary of the mean and standard deviation of the measurements made by conventional intraoral radiography (IOPAR) and ultrasound (US)

\begin{tabular}{lccc}
\hline Dimension & Technique & $\begin{array}{c}\text { Mean measurement } \\
(\mathrm{mm})\end{array}$ & Standard deviation \\
\hline Superoinferior & IOPAR & 12.50 & 3.17 \\
& US & 9.49 & 4.05 \\
Mesiodistal & IOPAR & 11.33 & 4.32 \\
& US & 12.08 & 4.83 \\
Anteroposterior & US & 9.14 & 3.71 \\
\hline
\end{tabular}

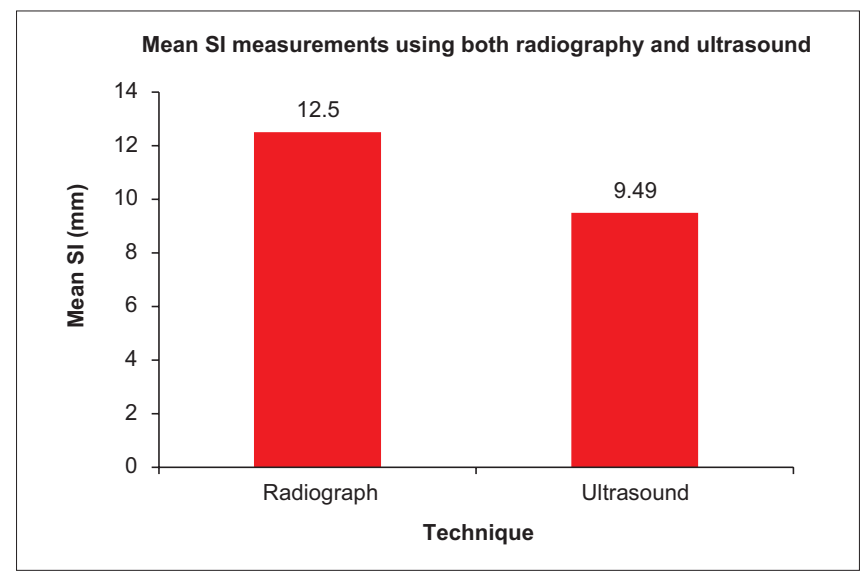

Figure 3: Mean superoinferior (SI) measurements with both conventional radiography and USG cyst with healing granulation tissue at the periphery. The remaining five were completely hypoechoic.

The other 10 lesions showed uniformly hypoechoic areas or mixed hypoechoic (in the center) and hyperechoic (in the periphery) areas with mildly irregular walls and the presence of internal vascularity on both color Doppler and power Doppler; these were diagnosed as granulomas.

USG measurements were made in all the three dimensions. The mean for superoinferior, mesiodistal, and anterosuperior dimensions were $9.49 \mathrm{~mm}$ (standard deviation: 4.05), 12.08 mm (standard deviation: 4.83), and $9.14 \mathrm{~mm}$ (standard deviation: 3.71), respectively [Table 1, Figure 3].

Comparison of sizes of periapical lesions on conventional radiography and USG

Statistical analysis showed no significant difference in the mesiodistal measurements obtained by conventional radiographs and by USG examination ( $t=0.633 ; P=0.5286)$. However, the superoinferior measurements showed significant difference $(P<0.01)(t=3.2055 ; P=0.0022)$ [Table 1, Figure 3].

\section{Histopathological examination}

Fifteen of 21 lesions diagnosed as periapical cyst on conventional radiological examination were confirmed as periapical cyst on histopathological examination. The remaining six were found to be periapical granuloma. Five out of nine lesions diagnosed as periapical granuloma on conventional radiological examination were confirmed as periapical granuloma on histopathological examination. The remaining four were found to be periapical cysts.

Nineteen out of 20 lesions diagnosed as periapical cyst on USG examination were confirmed as periapical cyst on histopathological examination, while one turned out to be a periapical granuloma. All 10 lesions diagnosed as periapical granulomas on USG examination were confirmed as periapical granuloma on histopathological examination [Table 2].

Comparison of conventional radiography and USG

Conventional radiography diagnosed periapical cyst with a sensitivity of $78.95 \%$ and a specificity of $45.55 \%$ and periapical granuloma with a sensitivity of $45.45 \%$ and a specificity of $78.95 \%$. In comparison, USG with color Doppler and power Doppler diagnosed periapical cyst with a sensitivity of $100 \%$ and specificity of $90.91 \%$ and periapical granuloma with sensitivity of $90.91 \%$ and specificity of $100 \%$.

The difference between the accuracy of radiographs and USG for the detection of periapical cyst (i.e., 66.67\% vs. 96.67\%) proved to be highly significant $\left(\chi^{2}=30.06\right.$; $\left.\mathrm{df}=1 ; P<0.001\right)$. Similarly, the difference between the accuracy of radiography 
Table 2: Summary of data comparing three diagnostic methods: conventional intraoral radiography, ultrasound imaging, and histopathology

\begin{tabular}{|c|c|c|c|c|c|c|}
\hline Patient & Age & Sex & Tooth & $\begin{array}{c}\text { Radiography } \\
\text { diagnosis }\end{array}$ & $\begin{array}{c}\text { USG } \\
\text { diagnosis }\end{array}$ & $\begin{array}{c}\text { Histo- } \\
\text { pathological } \\
\text { diagnosis }\end{array}$ \\
\hline 1 & 20 & $\mathrm{~F}$ & 12 & PC & PC & PC \\
\hline 2 & 13 & $\mathrm{M}$ & 21,22 & PC & $P G$ & PG \\
\hline 3 & 21 & $F$ & 21 & PC & IPC & IPC \\
\hline 4 & 22 & $\mathrm{M}$ & 42 & PG & IPC & IPC \\
\hline 5 & 21 & $\mathrm{~F}$ & 23 & PC & IPC & IPC \\
\hline 6 & 18 & $\mathrm{~F}$ & 21 & PG & $P G$ & $P G$ \\
\hline 7 & 16 & $\mathrm{M}$ & 22 & PC & IPC & IPC \\
\hline 8 & 18 & $\mathrm{M}$ & 21 & PC & PG & $P G$ \\
\hline 9 & 12 & $\mathrm{M}$ & 21,22 & PC & PC & PC \\
\hline 10 & 13 & $\mathrm{M}$ & 31,41 & PC & IPC & IPC \\
\hline 11 & 30 & $\mathrm{~F}$ & 11,21 & PC & $P G$ & $P G$ \\
\hline 12 & 32 & $\mathrm{~F}$ & 21 & PC & PG & PG \\
\hline 13 & 23 & $\mathrm{M}$ & 31 & PG & $\mathrm{PC}$ & PG \\
\hline 14 & 48 & $\mathrm{M}$ & 11 & PG & PC & PC \\
\hline 15 & 28 & $\mathrm{M}$ & 11 & PC & IPC & IPC \\
\hline 16 & 14 & $\mathrm{~F}$ & 11 & PG & PG & $P G$ \\
\hline 17 & 20 & $\mathrm{~F}$ & 21 & PC & $1 \mathrm{PC}$ & IPC \\
\hline 18 & 20 & $\mathrm{~F}$ & 21 & PC & IPC & IPC \\
\hline 19 & 21 & $\mathrm{M}$ & 21,22 & PC & $P G$ & $P G$ \\
\hline 20 & 22 & $\mathrm{M}$ & 11 & PG & $P G$ & $P G$ \\
\hline 21 & 43 & $\mathrm{M}$ & 11 & PC & IPC & IPC \\
\hline 22 & 22 & $\mathrm{M}$ & 21 & $P G$ & $\mathrm{PC}$ & $\mathrm{PC}$ \\
\hline 23 & 12 & $\mathrm{~F}$ & 41 & PG & IPC & IPC \\
\hline 24 & 35 & $\mathrm{~F}$ & 13 & PG & $P G$ & $P G$ \\
\hline 25 & 50 & $F$ & 22 & PC & IPC & IPC \\
\hline 26 & 23 & $\mathrm{M}$ & 11,12 & PC & IPC & IPC \\
\hline 27 & 19 & $\mathrm{~F}$ & 11 & PC & $\mathrm{PC}$ & $\mathrm{PC}$ \\
\hline 28 & 19 & $F$ & 11 & PC & $P G$ & $P G$ \\
\hline 29 & 23 & $\mathrm{M}$ & 11,12 & PC & $\mathrm{PC}$ & PC \\
\hline 30 & 32 & $\mathrm{M}$ & 11 & PC & PC & PC \\
\hline
\end{tabular}

PC: Periapical cyst, PG: Periapical granuloma, IPC: Infected periapical cyst

and USG for detection of periapical granuloma (i.e., $66.67 \%$ vs. $96.67 \%)$ proved to be highly significant $\left(\chi^{2}=30.06\right.$; $\mathrm{df}=1$; $P<0.001$ ) [Figure 4].

\section{Discussion}

USG provides specific information on the contents of the lesions. In the dental literature only three group of researchers, Cotti et al. in 2002 ${ }^{[6]}$ and 2003, ${ }^{[5]}$ Gundappa et al. in 2006, ${ }^{[8]}$ and Agarwal in 2008, ${ }^{[1]}$ have reported the use of USG in the examination of bone lesions of endodontic origin. Most of these studies are on small populations.

In this study we have used a bigger sample to evaluate the difference between USG with color Doppler and power Doppler and conventional bisecting-angle intraoral

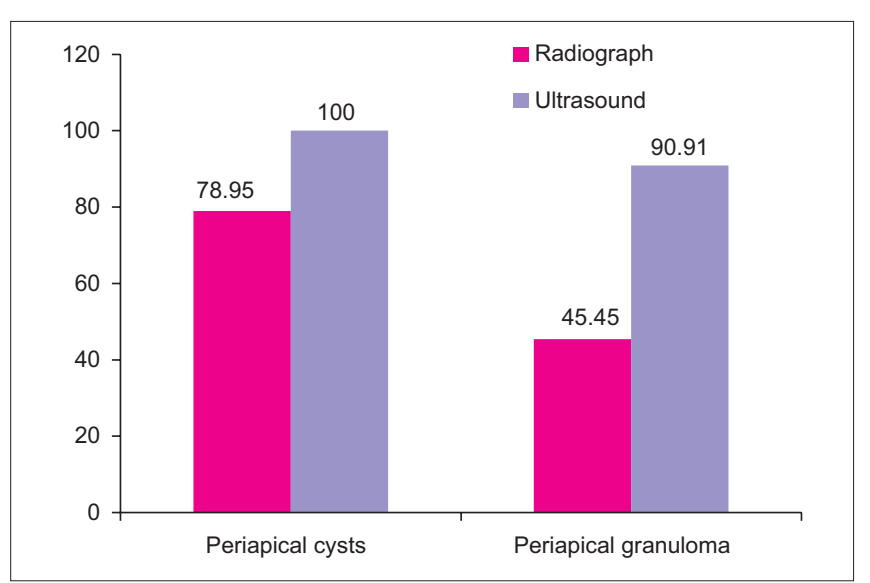

Figure 4: Comparison of the sensitivity and specificity of radiography and ultrasound in diagnosing periapical cysts and granulomas

periapical radiography in the diagnosis of periapical lesions.

All the previous studies have demonstrated a definite correlation between the echotexture of the lesions as well as their vascularity status and the histopathological features. Cotti et al. in $2003^{[5]}$ did a similar study in 11 patients and obtained 100\% agreement between USG and histopathology in all their cases. Similarly, Gundappa et al. in $2006^{[8]}$ found USG diagnosis to be $100 \%$ accurate in periapical lesions in a sample size of 15 patients.

In the present study, both the nature and the size of periapical lesions were evaluated on both conventional radiographs and USG. USG identified the contents and nature of 29 periapical lesions out of 30 lesions ( 19 cysts and 10 granulomas), whereas conventional intraoral radiograph identified only 21 lesions. The USG diagnosis agreed with the histopathological diagnosis in 29 cases. Thus, there was definite correlation between the echotexture of the lesions and the histopathological features except in the case of one lesion, which may have been due to some surgical error or processing error during histopathological procedures or due to the presence of a secondary infection in the lesion.

Traditionally, the size of a periapical radiolucent lesion has been used to differentiate between cyst and granuloma. Previous studies showed USG measurements to be smaller than corresponding radiographic measurements. In the present study, the mean superoinferior and mesiodistal measurements on USG examination $(9.49 \mathrm{~mm}$ and 12.08 $\mathrm{mm}$, respectively) were less than those on conventional radiography (12.50 $\mathrm{mm}$ and $11.33 \mathrm{~mm}$, respectively), consistent with previous studies. However, the mean mesiodistal measurement was slightly more on USG than on conventional radiography. This may have been because the bony edges of the lesions cast an acoustic shadow on the lateral walls, making it difficult to make exact measurements. ${ }^{[8]}$ 
USG examination in this study is limited to the anterior aspects of the jaws as the presently available probes are not ideal for use in the posterior jaws in areas of thick cortical plates. Further research is required for the development of suitable probes for the posterior jaws.

\section{Conclusion}

USG with color Doppler and power Doppler is superior to conventional intraoral radiographic methods for diagnosing the nature of periapical lesions in the anterior jaws.

\section{References}

1. Aggarwal V, Logani A, Shah N. The evaluation of computed tomography scans and ultrasounds in the differential diagnosis of periapical lesions. J Endod 2008;34:1312-5.

2. Bender IB, Seltzer S. Roentgenographic and direct observation of experimental lesions in bone. J Am Dent Assoc 1961;87:708-16.

3. Bender IB, Seltzer S. Roentgenographic and direct observation of experimental lesions in bone. J Am Dent Assoc 1961;87:708-16.

4. Sumer AP, Danaci M, Ozen Sandikçi E, Sumer M, Celenk P. Ultrasonography and doppler ultrasonogrsaphy in the evaluation intraosseous lesions of the jaws. Dentomaxillofac Radiol 2009;38: 23-7.

5. Cotti E, Campisi G, Ambu R, Dettori C. Ultrasound real-time imaging in the differential diagnosis of periapical lesions. Int Endod J 2003;36:556-63.

6. Cotti E, Campisi G, Garau V. A new technique for the study of bone lesions. Ultrasound real time imaging. Int Endod J 2002;35:148-52.

7. White SC, Pharoah MJ. In: Oral Radiology, principles and interpretation. $5^{\text {th }}$ ed. St. Louis, Missouri: Mosby Publication; 2004. p. 256-70, 338-54.

8. Gundappa M, NgSY, Whaites EJ. Comparison of ultrasound, digital and conventional radiography in differentiating periapical lesions. Dentomaxillofac Radiol 2006;35:326-33.

Cite this article as: Goel S, Nagendrareddy SG, Raju MS, Krishnojirao DRJ, Rastogi R, Mohan RPS, et al. Ultrasonography with color Doppler and power Doppler in the diagnosis of periapical lesions. Indian J Radiol Imaging 2011;21:279-83.

Source of Support: Nil, Conflict of Interest: None declared. 